CUAD. CONTAB. / BOCOTÁ, COLOMBIA, 15 (39): 745-762 / NÚMERO ESPECIAL 2014 / 745

\title{
Formulación de un tablero de comando para el análisis de la productividad y la gestión competitiva en el sector salud*
}

doi: 10.11144/Javeriana.cc15-39.ftca

\section{Eduardo Montico-Riesco}

Contador público. Licenciado en administración, Universi-

dad de la República Oriental del Uruguay.

Profesor invitado, Unidad de Perfeccionamiento de Egresados de la Universidad de la República. Profesor invitado, Instituto de Estudios Empresariales de la Universidad de Montevideo. Miembro del Colegio de Contadores Economistas y Administradores del Uruguay.

Correo electrónico: eduardomontico@gmail.com

\author{
Martín Velarde \\ Contador público, Universidad de la República Oriental \\ del Uruguay. MBA con énfasis en costos y gestión empre- \\ sarial (tesis en curso), Universidad de la República Orien- \\ tal del Uruguay. Profesor grado 2 en contabilidad de costos \\ y contabilidades especiales. Docente de posgrado de costos \\ en empresas de salud. Miembro del Colegio de Contadores \\ Economistas y Administradores del Uruguay. \\ Correo electrónico: mvelarde@adinet.com.uy
}

\footnotetext{
* El objetivo de este trabajo es La formulación de un tablero de comando para el sector salud que, por medio de métricas e indicadores, permita gestionar las empresas prestadoras de servicios de salud con énfasis en la productividad y costos. Este artículo corresponde a una versión mejorada de documentos publicados anteriormente, especialmente en la Revista del Instituto Argentino de Profesores Universitarios de Costos. Costos y Gestión 87
} 
Resumen El sector salud está sometido en la actualidad a una presión constante, debido a la demanda creciente, los costos en aumento y la verdadera invasión de nuevas tecnologías y fármacos puestos a disposición de los profesionales médicos día a día. A su vez, el envejecimiento de la población y la prevalencia de enfermedades crónicas, como la diabetes, el asma, la hipertensión arterial, etc. hacen que aumenten los promedios de estadía de los pacientes internados, que estos resulten más complejos y que el costo de su atención crezca en forma desmedida. Asimismo, la regulación por parte de las autoridades sanitarias impone un marco normativo que condiciona el accionar de este tipo de empresas. Esta conjunción de factores hace que resulte extremadamente complejo gestionar las empresas sanitarias y que sea necesario contar con instrumentos que permitan monitorear el conjunto de actividades realizadas por cientos y a veces miles de profesionales que interactúan entre sí, en general de una forma no coordinada. El objetivo de este trabajo es proponer una herramienta de gestión que brinde a los gestores de salud un conjunto de indicadores que permitan evaluar y eventualmente corregir los procesos asistenciales para alcanzar los planes estratégicos de la compañía midiendo la productividad y haciendo a estas empresas más competitivas.

Palabras clave gestión; productividad; tablero de comandos; competitividad

\section{Código JEL D 61, M 41}

\section{Formulating a Balanced Scorecard for Productivity Analysis and Competitive Management in the Health Sector}

\footnotetext{
Abstract Currently the health sector is under constant pressure due to the growing demand, cost increase, and a real invasion of new technologies and pharmaceuticals made available every day to the health professionals. At the same time, the ageing of the population and the prevalen-
}

ce of chronic illnesses, such as diabetes, asthma, arterial hypertension, etc., causes an unchecked increase in the average stay time of admitted patients to increase, as well as in the complexity of each case and the cost of their care. Likewise, the regulations from Health Authorities impose a normative framework that conditions the actions of this kind of companies. This combination of factors makes extremely difficult to manage health companies and makes necessary to have tools that allow the monitoring of all the activities performed by hundreds, even thousands, of professionals interacting between each other, generally in a non-coordinated way. The purpose of this work is to propose a management tool that gives the managers of the health sector a set of indicators, allowing them to assess, and eventually correct, medical care processes; in this way the strategic plans of the company can be achieved, measuring productivity, and making these companies more competitive.

Keywords management; productivity; balanced scorecard; competitiveness

\section{A teoria dos custos-desempenhos ocultos: abordagem teórica}

Resumo O documento faz aproximação teórica do conceito de custos ocultos, entendendo-os como os disfuncionamentos resultantes da interação entre estruturas organizacionais e comportamentos humanos. Tais custos são nomeados de ocultos pois não conseguem ser evidenciados pelos sistemas contábeis tradicionais por não terem as características dos custos visíveis, o que faz com que a sua existência e quantia seja ignorada na hora de tomar a maioria de decisões organizacionais. Os professores franceses Henri Savall e Véronique Zardet junto com o seu grupo de estudos do Institut de socio-économie des entreprises et des organizations (Instituto de Socioeconomia das Empresas e as Organizações, ISEOR) da Universidade de Lyon, trabalharam no desenvolvimento de um método que permitisse a medição de custos ocultos. Para isso, geraram 
uma série de indicadores com que procura-se determinar e eliminar os custos ocultos existentes numa organização. A partir da análise de custos-desempenhos ocultos, Savall e Zardet desenharam dois modelos administrativos: o Modelo de Gestão Socioeconómica que permite associar o desempenho económico da empresa (técnico e comercial) e o social e humano, e o Modelo HORIVERT (HORIzontalVERTical) que propõe melhorar a qualidade integral das organizações (interna e externa) e incrementar o desempenho económico e social. Este artigo visa ser base de futuras pesquisas sobre a temática ao mostrar — através da abordagem teórica-como é que esses modelos permitem às organizações obter maiores lucros sim necessidade de realizar mais investimentos nelas, o que melhora a gestão organizacional.

Palavras-chave contabilidade de gestão; custos; custos ocultos; desempenhos ocultos; disfuncionamentos

\section{Introducción}

Iniciaremos la introducción a este trabajo, con un breve diálogo extraído del clásico infantil Alicia en el país de las maravillas, de Lewis Carroll (1865).

Alicia: ¿Podría decirme, por favor, qué camino he de seguir desde aquí?

Gato de Cheshire: Eso depende en buena medida del lugar adonde quieras ir.

Alicia: No me importa mucho a dónde...

Gato de Cheshire: Entonces no importa por dónde vayas.

Este es un conocido ejemplo en los textos de planeamiento estratégico y trasluce la importancia de tener claro hacia dónde se mar- cha... en el país de las maravillas o en el mercado.

Si no se tienen claros los objetivos y planes estratégicos, y si no se sabe hacia dónde se dirige la compañía, no se puede gerenciar la empresa por el camino del éxito; en suma, no se la puede conducir hacia el cumplimiento de la misión y demás objetivos estratégicos.

Ahora bien, ¿qué implica esto?

En primer lugar, una definición clara de la misión de la empresa, de sus objetivos, planes y metas estratégicas acorde al plan estratégico de la compañía, esto es, hacia dónde quiero dirigirme, pero a su vez... tener claro dónde me encuentro y cuál es el camino por el que estoy transitando para llegar al destino deseado. En suma, debo contar con instrumentos que me permitan medir la posición actual en la que se encuentra la empresa y cómo esta se acerca o no a las metas trazadas.

O sea, debo contar con un conjunto de instrumentos de medición que me indiquen en forma permanente dónde estoy, por dónde voy y si mi acerco o me alejo del destino trazado. Es decir, debo contar con un GPS de gestión...

Debemos contar entonces con un tablero de control que mida diferentes perspectivas de la empresa en el logro de los planes estratégicos, en la literatura abundan múltiples ejemplos de este tipo de instrumentos. Uno de los más conocidos es el Tablero de Comando o Balanced ScoreCard, BSC, de los profesores Robert Kaplan, de la Universidad de Harvard y David P. Norton, presidente de Nolan, Norton \& Company (Kaplan \& Norton, 1992). En este trabajo, propondremos otro enfoque dirigido al sector salud. 


\section{Indicadores: definición y características}

$\mathrm{Al}$ construir el tablero de control, es preciso definir un conjunto de instrumentos o indicadores que me permitan medir, como ya dijimos, si las acciones que lleva adelante la compañía están alineadas con mis metas y objetivos.

Como este trabajo está orientado al sector salud, veremos una de las definiciones de indicador según el Glosario de términos, de la Organización Mundial de la Salud, OMS (s.f.):

\begin{abstract}
Variable con características de calidad, cantidad y tiempo, utilizada para medir, directa o indirectamente, los cambios en una situación y apreciar el progreso alcanzado en abordarla. Provee también una base para desarrollar planes adecuados para su mejoría.
\end{abstract}

La misma se adapta perfectamente al objetivo propuesto, mide los cambios en la situación, aprecia el progreso y nos permite desarrollar planes para mejorar la situación en caso de no estar en el camino acertado.

Ahora bien, definido qué entendemos por indicador, es necesario establecer cuáles son elementos que deben componer un indicador, a saber:

- Nombre o denominación. Esto es la identificación clara y precisa del indicador, la misma es necesaria a los fines de que se pueda descubrir la característica o hecho que se medirá.

- Propósito. Expresa el para qué, esto es, cuál es el motivo de calcular el indicador definido y qué se desea medir con él.
- Patrón de comparación. El indicador será comparado con un estándar o un patrón de referencia definido previamente para establecer si hay o no desviación. Hay diversos patrones de comparación o niveles contra los cuales se comparará el indicador, como el nivel histórico, el teórico, el nivel de requerimiento de los usuarios, el nivel de competencia, el nivel de planeación, etc. Se define entonces un objetivo o meta para el indicador.

- Fórmula para el cálculo. Es el mecanismo para determinar el valor del indicador. En el caso de indicadores cuantitativos, se debe precisar con claridad la fórmula con la cual se realizará el cálculo de su valor. Es decir, se identificarán en ella los factores que se relacionan con las unidades en las cuales se expresa el valor del indicador.

- Vigencia. En entornos cambiantes, los indicadores deben ser revisados periódicamente para que la información suministrada por estos sea pertinente y oportuna. Esto quiere decir que se deben actualizar tomando en consideración los cambios del entorno y las necesidades reales de información de la institución para no agotar esfuerzos ni gastar recursos en calcular indicadores que no generan valor agregado a la institución.

- Valor agregado. El indicador debe reportar utilidad a la institución para la toma de decisiones, es decir, debe tener una razón de ser que amerite su construcción y posterior seguimiento.

- Periodicidad. Al establecer un indicador se debe especificar el período en el que se generará, es decir, se debe señalar cada cuánto 


\section{TABLERo de COMANDo EN EL SECTOR SALUd / E. MONTICO, M. VelaRde / 749}

tiempo el nivel responsable debe realizar la medición para calcular el indicador.

Ahora bien, los indicadores a su vez deben poseer un conjunto de características propias, a saber:

- Validez: el indicador debe reflejar el aspecto para el que se creó o estableció y no otro.

- Confiabilidad: debe brindar el mismo resultado en iguales circunstancias.

- Comprensibilidad: debe comprenderse fácilmente qué aspecto pretende reflejar.

- Sencillez: debe ser sencillo de administrar, de aplicar y de explicar.

Estas características están alineadas con los conceptos de lean management y del gerenciamiento visual, que por medio de indicadores de alto contenido "visual" transmiten claramente el lenguaje de los procesos de negocios: cómo estamos, en qué sentido vamos, etc.

\section{Sector salud. Características}

Previo a la construcción del modelo propuesto, es necesario establecer determinadas características del sector salud que se deberán tener en cuenta a la hora de definir los indicadores y el tablero de comando propuesto en este trabajo.

Podemos decir que los servicios prestados en una empresa de salud presentan las siguientes características (Montico-Riesco \& Velarde, 2003):

- Intangibilidad: aunque hay elementos materiales como placas, radiografías, insumos, etc., el producto más importante que brinda es la atención y/o intervención médica, la cual no se puede materializar físicamente.

- Unidad de medida: es complejo establecer la cantidad de servicios producidos, dada su heterogeneidad.

- Fabricación artesanal: no se pueden automatizar como en las industrias, a pesar de que se está avanzando en cirugía robótica y seguramente en un futuro, esto pueda cambiar.

- Heterogeneidad: por ejemplo, no es lo mismo realizar una cirugía cardiaca a una persona que hacerle una operación en la vista. Los recursos utilizados y los resultados obtenidos en dos personas a las que se les hacen cirugías cardiacas pueden ser diferentes; de hecho, lo son.

Entonces se presenta el primer problema importante: la definición de cuál es el producto o servicio brindado, y sobre todo cómo lo podemos medir. Natividad Guadalajara (1994) ofrece la primera visión sobre el tema cuando menciona que un servicio de salud se compone de la prestación principal y de unas prestaciones secundarias. Por ejemplo, una prestación principal es una cirugía cardiaca mientras que la prestación secundaria es la atención al enfermo, materiales y medicamentos utilizados, limpieza, comida, etc.

Así como Natividad Guadalajara (1994) dividió al servicio prestado de salud en prestación principal y secundaria, se puede dividir también en servicio intermedio y final.

En una institución de asistencia médica, hay muchos productos/servicios, unos físicos y otros intangibles (la mayoría). Bajo la clasificación 
mencionada en el párrafo anterior, el conjunto de todos estos es el de productos intermedios. El producto final es el conjunto de productos intermedios brindados a cada paciente.

Si queremos avanzar un poco más, hay servicios intermedios que se pueden clasificar como de apoyo: los departamentos de mantenimiento, cocina, lavadero, etc., que si bien no se prestan directamente al paciente son necesarios para que se brinde el servicio intermedio y, por ende, el servicio final.

Hay determinados productos de salud, intangibles (atención médica, diagnóstico, etc.) y tangibles (análisis clínicos, placas radiológicas, etc.) que con el apoyo necesario se combinan de tal manera que cada combinación se puede definir como un servicio final.

Esto da cuenta de la dificultad y/o particularidad del cálculo de costos en estas instituciones y, por ende, de medir la productividad y eficiencias como veremos más adelante.

Hay que recordar por ejemplo que para obtener una placa, previamente se necesitó el mantenimiento de la máquina que hace las placas, la limpieza de la sala de placa y del consultorio, todos estos servicios de apoyo.

A su vez, el producto/servicio presentará una gran variedad, debido a la diversidad de pacientes tratados, entonces no solo tenemos muchos productos, sino que, a su vez, cada producto es muy variable de acuerdo al paciente tratado.

En resumen, nos enfrentamos a una actividad con sus complejidades propias, con gran disparidad de recursos utilizados y, por ende, con productos dispares.

\section{El objeto de costos en salud}

Como hemos mencionado en otros trabajos, es extremadamente importante determinar cuál es el "objeto" de costo en salud. Para nosotros, el objeto de costo es, sin duda, el paciente; pero no el "paciente promedio" sino que se debe costear de acuerdo a su patología, porque la misma es determinante a la hora de agrupar y analizar las diferentes ópticas que se analizarán.

En suma, todo análisis de las distintas variables que se determinen por medio de los diversos indicadores que veamos, deben tener como línea de corte la patología del paciente, no es viable hacer una comparación entre diversos indicadores si no se tiene en cuenta este aspecto.

\section{Promedios, ¿una medida válida en salud?}

De cara a estas definiciones, y frente a la propuesta de cálculo de diversos indicadores que tengan por objetivo constituirse en una guía de acción, sobreviene la pregunta sobre si en el sector salud es válido calcular indicadores globales que en definitiva toman en cuenta por ejemplo la sumatoria de recursos utilizados y la comparan con la producción obtenida, lo que arroja promedios globales.

Desde nuestro punto de vista, la respuesta es NO. El promedio estadístico de una población es una medida de resumen que intenta mostrar un valor "medio". Este promedio intenta representar mediante ese valor, al conjunto de la población. Esta medida de resumen es válida como medida representativa siempre y cuando la desviación 


\section{TABLERO DE COMANDO EN EL SECTOR SALUd / E. MONTICO, M. VELARDE / 751}

estándar no sea demasiado abultada, vale decir, cuando la "distribución" se concentra cercanamente a la media población.

Si la población sujeta a análisis tiene valores extremadamente dispersos, el cálculo de la media poblacional no aportará demasiado valor para su análisis. A modo de ejemplo, ¿qué quiere decir que el promedio entre 1 y 100 es
50,5?... No mucho, pues no representa ni a uno ni otro.

Por tanto, cuando queramos tomar como indicador un promedio, debemos asegurarnos de que este está relacionado a productos/servicios homogéneos. Veamos el siguiente ejemplo, que ya hemos expuesto en otros trabajos pero que es clarificador:

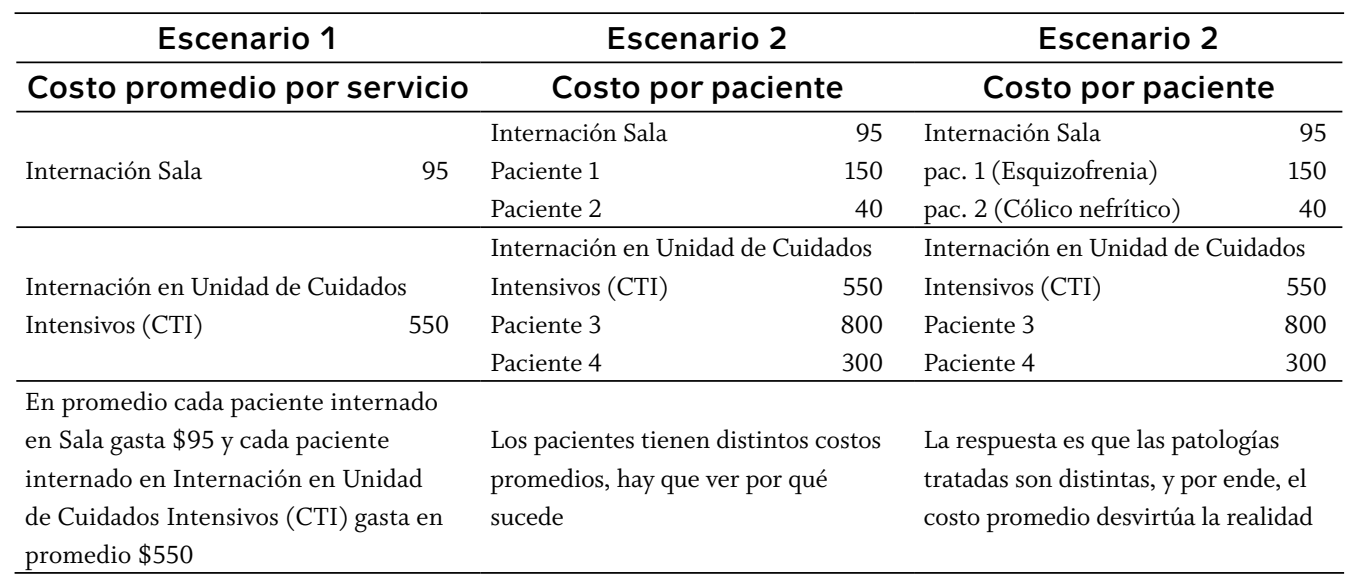

Tabla 1

Comparativo sistemas de costeo

Fuente: elaboración propia

Este ejemplo de promedios está referido a costos promedios de día/cama, y en el mismo se puede apreciar que la información del escenario 1 es tan genérica que - creemos-desvirtúa el promedio. ¿Por qué? Porque los productos/ servicios que estamos usando para calcular el indicador son heterogéneos.

Esta apreciación - que puede parecer evidente-es muy importante, ya que de nada serviría calcular indicadores, si no se tiene en cuenta que el valor arrojado puede no ser representativo de la situación que se pretende analizar.

\section{Indicadores y ratios. Semejanzas y diferencias}

En la práctica, muchas personas hablan indistintamente de ratios o indicadores como si fueran lo mismo, pero en nuestra opinión las ratios y los indicadores pueden estar muy relacionados entre sí o ser muy distintos, dependiendo de qué ratios y de qué indicadores estemos hablando. Veámoslo.

Una ratio es una razón, es decir, un cociente entre dos magnitudes o cifras relacionados entre sí y expresados en la misma unidad de 
medida (moneda o unidades físicas). Muestra la relación que hay entre los dos términos comparados y se expresa tanto en cifras como en porcentajes.

Los indicadores, sin embargo, son parámetros que se utilizan para el seguimiento de determinado objetivo. La idea es que mediante un continuo análisis de los indicadores en el tiempo se vaya viendo si se están cumpliendo los objetivos planteados.

En otras palabras, un indicador nos permite ir evaluando si se alcanzó o no un objetivo. Es decir, tengo que fijarme un objetivo (en cualquier ámbito y a cualquier nivel) y a partir de él, ir chequeando la realidad para ver si lo consigo. Ese chequeo de la realidad lo hago mediante indicadores.

Algunos indicadores pueden ser ratios, pero otros pueden ser solamente algún aspecto específico de la información. Por ejemplo, si la empresa se fija el objetivo de aumentar en tres años las ganancias actuales en un 50\%, el indicador que podremos ir chequeando es la ratio de rentabilidad sobre ventas. En este caso, el indicador es una ratio. Pero también podemos tomar como indicador la ganancia del ejercicio y ver su evolución a través de los meses o los años; aquí, el indicador no es una ratio sino una sola variable extraída del sistema de información contable.

Para que un indicador pueda ser útil lo ideal sería, como dijimos antes, que estuviera relacionado con un objetivo empresarial específico. Sin embargo, las ratios por sí solas sirven para analizar situaciones particulares, que pueden ser comparables en el tiempo para ver su evolución (si la situación mejora o empeora), pero no ne- cesariamente tiene que estar relacionado con un objetivo específico más que el análisis de tal situación. Ellos constatan realidades. Veamos otro ejemplo de cómo una ratio puede no ser un indicador y valerse sola como ratio en un análisis; y cuándo se transforma en un indicador.

Una ratio muy utilizada en los análisis de los estados contables es la de la razón corriente, calculada como la división el activo corriente y el pasivo corriente. Esta ratio es una medida de liquidez que nos muestra la proporción de deudas de corto plazo que la empresa puede pagar con los elementos del activo que se convertirán en efectivo en menos de un año. La ratio indica entonces que, si es mayor que 1 , los activos corrientes de la empresa teóricamente pueden cancelar todos aquellos pasivos; y si es menor que 1 , lo contrario, es decir, la empresa va a tener que salir a buscar fondos o suspender pagos.

Esto se desprende de la ratio y nada más (ni nada menos); podemos, si queremos, compararla con años anteriores y ver su evolución, pero ya cumplió su función como ratio.

La situación es diferente si en su planificación estratégica la empresa se ha planteado que para conseguir los objetivos generales debe tener una razón corriente mayor a 1,2. Aquí, si bien la información de la ratio es la misma, como vimos antes, la ratio es un indicador de si se está cumpliendo el objetivo fijado; así que si la ratio no es mayor a 1,2, la empresa tratará de mejorarlo. En el caso anterior, quizás también tratará de mejorarlo, pero no porque se haya fijado un objetivo determinado.

Tal es la relación entre una ratio y un indicador. En nuestra propuesta, hablaremos de indicadores. 
Una pregunta importante para su diseño es: ¿quién debe manejarlos?, ¿solo la gerencia y la dirección? No, las ratios e indicadores deben ser manejados no solo por ellos sino también por los jefes de los servicios asistenciales, pues en general cada servicio tiene un encargado. Lo que diferencia el cuadro de la Gerencia del cuadro del Jefe de Servicio es el alcance. Mientras los primeros miran los indicadores y las ratios más generales de la actividad, los segundos miran la evolución de los más específicos.

\section{Propuesta de cuadro de mando}

Una vez realizada esta precisión respecto a la importancia de la patología de los pacientes como elemento de corte a la hora de realizar comparaciones, propondremos un tablero de comando que contendrá un conjunto de indicadores de gestión que permitan el seguimiento de las variables claves (KPI, Key Performance Indicators) de la empresa y sus diferentes métricas.

Los mismos contemplarán los diferentes aspectos definidos por Avedis Donabedian (2002), cuya propuesta conceptual está basada en tres elementos: estructura, proceso y resultado.

En este sentido, se analizará un conjunto de indicadores que involucren las prestaciones de servicios, sus costos y tengan en cuenta elementos de calidad, a saber:

- Vinculados a las prestaciones de asistencia.

- Vinculados a los costos y utilización de recursos asociados a las prestaciones de asistencia.

- Vinculados a la medición de la calidad.

Obviamente, dentro de cada uno de estos grupos hay una multiplicad de indicadores, que miden diversas perspectivas. La propuesta de este trabajo está en seleccionar aquellos indicadores que impacten en la productividad y mejoren la competitividad, que es su objetivo.

En primer término, creemos oportuno precisar el concepto de productividad en el área de la salud. La productividad se podría definir como la relación entre los recursos utilizados y los productos/servicios prestados, sin dejar de tener en cuenta la calidad. Por ende, no solamente definiremos indicadores que tiendan a medir la productividad e impacten en la competitividad, sino que integraremos mediciones de la calidad de los servicios.

En suma, la productividad tiene qué ver con los costos de las prestaciones de asistencia, o sea, el consumo de recursos y los productos/servicios que se obtienen.

\section{Indicadores vinculados a las} prestaciones de asistencia ambulatoria y de emergencia

Estos indicadores miden la productividad del recurso utilizado, ya sea humano o de las instalaciones físicas. A su vez, se analizarán los indicadores que tengan relación con su capacidad resolutiva.

Los indicadores que se mencionan a continuación se deben analizar desde diferentes ángulos, o sea, por médico, especialidad, etc. De lo contrario, estaríamos en la situación descrita en el apartado 4, Promedios, ¿̇una medida válida en salud?, o sea calculando promedios que pueden tener un grado de dispersión muy importante respecto a la media. Reiteramos que si queremos comparar dos indicadores entre sí, se deberá tener en cuenta la patología del paciente. 
Los indicadores propuestos en este sentido son:

\section{Denominación}

Propósito

Fórmula de cálculo

Este indicador mide cuántas consultas ambulatorias se realizan en un período dado, con los recursos humanos existentes y la planta física disponible.

\section{Denominación}

\section{Cantidad de consultas/mes}

Determinar el total de consultas efectuadas

Sumatoria de consultas totales en el mes

\begin{tabular}{lc}
\multicolumn{1}{c}{ Denominación } & Promedio consultas por médico/mes \\
\hline Propósito & Determinar cuántas consultas tiene un médico por mes \\
\hline Fórmula de cálculo & Sumatoria de consultas totales al mes/total de médicos \\
\hline $\begin{array}{l}\text { Este indicador mide cuántas consultas ambulatorias realiza cada médico en un período dado. Es un indicador típico de } \\
\text { medición de la productividad de cada médico; este indicador muestra claramente la importancia de la patología tratada, } \\
\text { no consume el mismo tiempo un paciente psiquiátrico que una consulta por un problema alérgico. }\end{array}$ \\
\hline
\end{tabular}

\section{Denominación}

Propósito

Fórmula de cálculo

\section{Tiempo promedio de reiteración de consulta}

Determinar el tiempo promedio en el cual el mismo paciente vuelve a ser visto en una consulta luego de la primera

Sumatoria de diferencia de tiempo entre la primera consulta y las sucesivas/total de pacientes vistos

Este indicador mide cuánto tiempo demora el paciente en volver a ser visto por el médico; es aplicable en aquellas patologías no crónicas que, por sí mismas, requieren reiterar periódicamente una consulta. Es un indicador muy fuerte de la capacidad de resolución de los problemas del paciente, si el médico debe reiterar de nuevo la consulta, seguramente no resolvió el problema de su paciente. Este indicador impacta fuertemente en la competitividad de la empresa, ya que si el paciente debe reiterar su consulta sin resolver su problema, esto sin duda afectará la imagen de la empresa.

\section{Denominación}

Propósito

Fórmula de cálculo

\section{Porcentaje de pacientes derivados a especialistas}

Determinar el porcentaje de los pacientes que son derivados a especialistas y no resueltos con el médico que los brindó consulta

Pacientes derivados a especialistas/sumatoria de pacientes vistos x 100

Este indicador posibilita analizar también la capacidad resolutiva del médico: un indicador demasiado alto mostrará que el médico no puede resolver los problemas de sus pacientes y que los deriva a los niveles superiores; esto genera descontento entre los pacientes que transitan por varios médicos sin resolver sus problemas y, por ende, demerita la imagen de la empresa.

\section{Denominación}

Propósito

\section{Cantidad de paciente vistos por hora}

Determinar la cantidad de consultas efectuadas por hora en cada especialidad Número de consultas por especialidad/cantidad de horas de la especialidad

Fórmula de cálculo Este indicador mide la productividad de cada especialidad, vale decir, cuántas consultas se "producen" por cada hora asignada. En general, la autoridad sanitaria establece un máximo de consultas por hora para que el médico dedique un tiempo prudencial a la atención del paciente. 


\section{TABlero de comando en El Sector SAlud / E. MONTICo, M. Velarde / 755}

\begin{tabular}{ll}
\hline Denominación & Tiempo de respuesta consulta de emergencia \\
\hline Propósito & $\begin{array}{l}\text { Determinar el tiempo promedio que transcurre desde que el enfermo demanda la asistencia o } \\
\text { llega a la unidad funcional, hasta que toma contacto con el médico o el equipo sanitario }\end{array}$ \\
\hline Fórmula de cálculo & Sumatoria de tiempos de respuesta/total de pacientes atendidos \\
\hline $\begin{array}{l}\text { Este indicador mide el tiempo de respuesta para la atención en emergencia: un indicador demasiado alto dará una } \\
\text { perspectiva negativa del aparato asistencial dedicado a la atención de emergencia y es un aspecto negativo que impactará } \\
\text { la competitividad frente a otros centros. }\end{array}$ \\
\hline
\end{tabular}

\section{Denominación Pacientes derivados a los niveles superiores}

\begin{tabular}{ll}
\hline Propósito & Determinar el número de pacientes que debieron ser derivados a un nivel superior de asistencia \\
\hline Fórmula de cálculo & Total pacientes derivados/total pacientes atendidos x 100
\end{tabular}

Este indicador posibilita analizar también la capacidad resolutiva del médico: un indicador alto mostrará que el médico no puede resolver los problemas de sus pacientes y que los deriva a los niveles superiores. Esto genera descontento entre los pacientes que transitan por varios médicos sin resolver sus problemas y, por ende, demerita la imagen de la empresa.

\section{Indicadores vinculados a las prestaciones de internación}

A continuación, veremos un conjunto de indicadores vinculados a la actividad de internación que enfocan también la productividad y la mejora de la competitividad.

\section{Denominación}

\section{Total de días de hospitalización/mes}

\begin{tabular}{ll}
\hline Propósito & Indica el total de días que estuvieron internados los pacientes egresados \\
\hline Fórmula de cálculo & Días camas ocupadas por pacientes egresados en el mes \\
\hline
\end{tabular}

Este indicador mide la cantidad de días de hospitalización en la estructura asistencial.

\begin{tabular}{ll}
\hline Denominación & Total de días egresos/mes \\
\hline Propósito & Indica el total de egresos del mes \\
\hline Fórmula de cálculo & Suma de egresos del período \\
\hline Este indicador mide la cantidad de egresos que se producen en la estructura asistencial.
\end{tabular}

Este indicador mide la cantidad de egresos que se producen en la estructura asistencial.

\section{Denominación}

\section{Promedio de estadía}

Propósito Indica la permanencia promedio de los pacientes hospitalizados

Fórmula de cálculo Total días camas ocupadas/total egresos

Este indicador mide cuánto tiempo permanece un paciente en promedio en el hospital. Se debe calcular vinculado a la patología del paciente, ya que las diferentes estadías dependen fuertemente de esta variable.

\begin{tabular}{ll}
\hline Denominación & \multicolumn{1}{c}{ Tasa de ocupación } \\
\hline Propósito & Mide el porcentaje de camas ocupadas en la estructura asistencial \\
\hline Fórmula de cálculo & Camas ocupadas/total camas disponibles \\
\hline $\begin{array}{l}\text { Mide el porcentaje de las camas sanatoriales que están ocupadas. Es un fuerte indicador del uso de la estructura y es } \\
\text { necesario calcularlo en función de la complejidad de las camas, las camas ocupadas, el cuidado convencional, el cuidado } \\
\text { crítico, etc. }\end{array}$
\end{tabular}


TABLERo de COMANDO EN EL SECTOR SALUd / E. MONTICO, M. VELARDE / 757

\begin{tabular}{ll}
\hline \multicolumn{1}{c}{ Denominación } & \multicolumn{1}{c}{ Largo de la cola de espera para consulta ambulatoria } \\
\hline Propósito & Mide la cantidad de días en espera de consulta \\
\hline Fórmula de cálculo & Fecha consulta - fecha solicitud \\
\hline
\end{tabular}

El mismo indicador se puede calcular para la coordinación de procedimientos quirúrgicos, a saber:

\begin{tabular}{ll}
\hline \multicolumn{1}{c}{ Denominación } & Largo de la cola de espera para coordinación quirúrgica \\
\hline Propósito & Mide la cantidad de días en espera de coordinación \\
\hline Fórmula de cálculo & Fecha coordinación - fecha solicitud \\
\hline
\end{tabular}

Los siguientes indicadores están muy vinculados a los resultados asistenciales

\begin{tabular}{ll}
\hline \multicolumn{1}{c}{ Denominación } & \multicolumn{1}{c}{ Tasa de mortalidad } \\
\hline Propósito & Mide la cantidad de pacientes fallecidos \\
\hline Fórmula de cálculo & Pacientes con egreso fallecidos/total egresos \\
\hline \multicolumn{1}{c}{ Denominación } & Tasa de reingresos \\
\hline Propósito & Mide la cantidad de pacientes reingresados \\
\hline Fórmula de cálculo & Pacientes con reingreso/total egresos \\
\hline & \\
\hline \multicolumn{1}{c}{ Denominación } & \\
\hline Propósito & Mide la cantidad de pacientes infectados \\
\hline Fórmula de cálculo & Pacientes infectados/total egresos \\
\hline
\end{tabular}

\section{Círculo CCC. ¿Podemos salir de él?}

Como hemos analizado en los capítulos anteriores de este trabajo, hemos definido un conjunto de indicadores que permiten medir los costos, tienen en cuenta la calidad y nos ayudan a visualizar cómo se afecta la competitividad.

Previo a analizar el concepto de Círculo CCC-Calidad-Costos-Competitividad-, debemos hacer un análisis preliminar de las instituciones prestadoras de salud, públicas o privadas.
Como mencionamos al principio de este trabajo, múltiples factores como el envejecimiento de población, la prevalencia de enfermedades crónicas, el surgimiento de nuevas tecnologías y medicamentos, el aumento de las demandas por mala praxis, etc., han reducido drásticamente los resultados operativos de estas empresas, con lo cual la variable clave resultado operativo no es una variable de ajuste de la cual se pueda echar mano.

El resultado operativo, en general, no tiene la magnitud necesaria para poder disponer de él como fuente de financiación de nuevas actividades o importantes inversiones. 
Por tal motivo, estos indicadores revelan una compleja situación de interacción de unos sobre otros, que hemos denominado el Círculo CCC. Esto quiere decir que la variación sobre uno de ellos puede impactar sobre los otros y generar un círculo vicioso del cual es difícil salir.

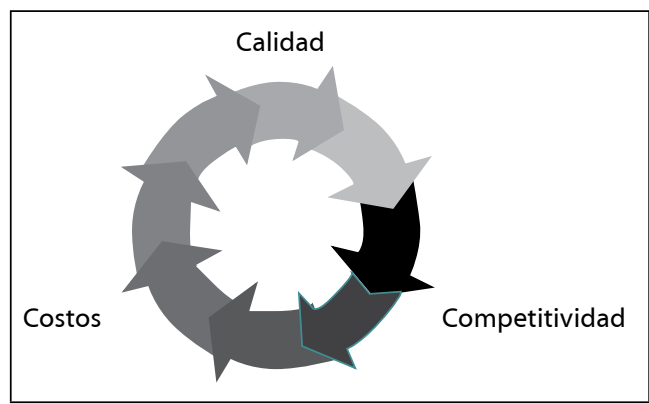

Gráfico 1

Círculo costo calidad competitividad Fuente: elaboración propia

Para simplificar este concepto, veamos el siguiente caso... Supongamos que la empresa está preocupada pues el largo de la cola de espera para consultas - uno de los indicadores de calidad más usuales - está aumentando; esto quiere decir que el tiempo promedio para ser atendido en una consulta se está prolongando en demasía.

En este caso, la empresa puede tomar la alternativa de asignar más médicos a la especialidad que el indicador muestra con valores elevados; esto generaría un aumento de los costos, lo cual — dada la restricción de un resultado operativo de bajo nivel-obligaría seguramente a reducir otro rubro, lo que seguramente haría bajar las prestaciones, caería la calidad y... ¡volveríamos a empezar de nuevo!!!
Otro ejemplo frecuente es el aumento de los promedios de estadía, lo que incrementa los costos de internación. Una alternativa es la de acelerar las altas de los pacientes, pero esta situación puede traer reingresos precoces, con lo cual tenemos menos estadías promedio, pero más días camas estadía, debido a los reingresos, por lo cual volvemos a aumentar el costo total.

Lo que estamos viendo es que esta situación limitante lleva a las decisiones sobre inversión y gastos a un "juego de suma cero", utilizando conceptos de la teoría de los juegos (Neumann \& Morgenstern, 1944), lo que aumenta un rubro de gastos inevitablemente deberá bajar otro. Esto produce en general un aumento de costos por otra vía o una pérdida de la "percepción" de la calidad que afecta de nuevo la competitividad.

Entonces... ¿¿no hay solución? SÍ las hay, aunque obviamente ninguna es sencilla. Dada nuestra experiencia en gestión de salud, proponemos por lo menos dos de ellas, a saber:

1. Lean Management: en general, no hay un estudio afinado en los procesos en las instituciones de salud. Su estudio permite frecuentemente reducir costos en muchas actividades y, por tanto, reasignar recursos en las áreas que necesitan una mejora de la calidad.

2. Teoría del valor: está muy vinculada con lo anterior, pero permite visualizar cuáles son aquellas actividades de no valor agregado para reducirlas al mínimo y obtener los recursos necesarios para destinar a las áreas problemáticas. En este análisis, la percepción del cliente respecto a los servicios brindados es de vital importancia: hay va- 


\section{TABLERO de COMANDO EN EL SECTOR SALUd / E. MONTICO, M. VeLARDE / 759}

rios "gaps" entre lo que el cliente quiere y lo que nosotros "entendemos" que él quiere. Un estudio afinado de esta perspectiva nos dirá hacia dónde asignar los recursos.

En suma, en la situación actual de recursos limitados, inversiones crecientes y demandas de servicios en alza, el análisis de indicadores permitirá focalizar nuestra atención en aquellos aspectos de la prestación en los que debemos mejorar nuestro desempeño y aplicar estrategias de mejora continua en los diversos procesos. Sin embargo, queda un problema por resolver que veremos a continuación.

\section{Herramientas de Business Intelligence. ¿Después de los indicadores, qué?}

Muchas empresas cometen el error de transformar el cuadro de indicadores en un fin de sí mismo, al focalizar todo el esfuerzo en obtener esos datos, sin percibir que ellos son en realidad el principio del análisis... una guía de cómo está marchando la compañía... si voy bien o voy mal...

Ahora bien, si todo está alineado con la estrategia corporativa, no hay problema... ¿Pero si los indicadores avanzan hacia zonas rojas?

¿Cómo determinar la causa o las causas de los desvíos cuando los objetivos estratégicos no se cumplen en un proceso altamente complejo como la prestación de asistencia sanitaria, en el cual intervienen médicos, técnicos, equipamiento, insumos, instalaciones, estudios paraclínicos y un sinnúmero de componentes?
¿Cómo corregir los desvíos, sin olvidar que este tipo de empresas posee un componente muy especial pues no solo es un tema de rentabilidad, ya que el servicio que presta está enfocado en el bien más preciado de un ser humano: la vida? Por ende, no corregir un problema a tiempo puede tener un costo no recuperable.

La tecnología da una respuesta posible. Hay herramientas informáticas capaces de analizar la información desde distintos ángulos, de profundizar en los procesos y de correlacionar las distintas variables para determinar las tasas de ocurrencia de cada una de ellas respecto a los eventos ocurridos.

Estas herramientas, denominadas de inteligencia de negocios, a partir de los tableros de comando, permiten navegar en profundidad y seccionar la información hasta sus componentes más íntimos. Mediante los conceptos de Data Warehousing, OLAP (On-Line Analytical Processing), Datamining y análisis predictivo, entre otros, estas técnicas facilitan encontrar las respuestas a las tres preguntas planteadas en el concepto de Corporate Performance Management (CPM), a partir del cual están diseñadas las principales soluciones disponibles y se busca la continuidad en el análisis preliminar que surge de la visualización de un indicador.

Este modelo busca potenciar la eficiencia que se puede obtener en el procesamiento de la información, que cada vez es mayor en las organizaciones, a partir del uso de diversas herramientas informáticas de gestión (sistemas ERP, CRM, etc.), en conjunto con la efectividad en el análisis que mejore la capacidad en la toma de decisiones. 


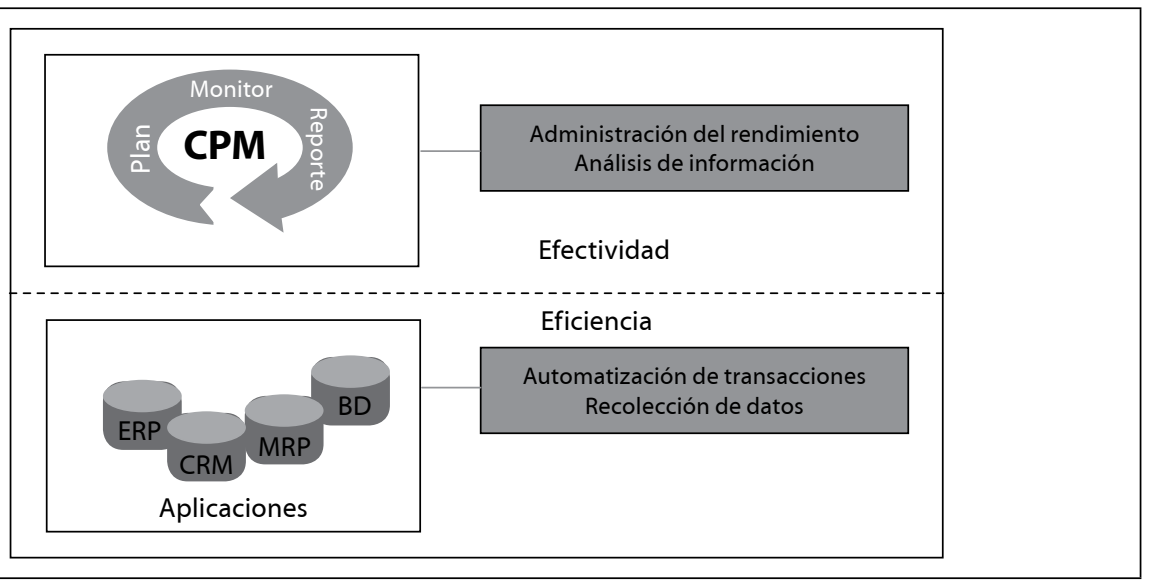

Gráfico 2

Herramientas para medir efectividad y eficiencia

Fuente: elaboración propia

De esta forma, las herramientas informáticas alineadas con estos conceptos buscan facilitar las respuestas a las siguientes preguntas:

\section{1. ¿Cómo estamos?}

Podemos responder esta pregunta mediante el conjunto de indicadores y métricas definidos en el Balanced ScoreCard, BSC, que indican los puntos de enfoque de la gestión, alineados a la estrategia.

Las herramientas informáticas actuales permiten, además de visualizar el comportamiento de los indicadores en relación con el objetivo definido, incorporar diversos instrumentos para complementar esta rápida visión de la situación, como:

\section{- Scorecards y Dashboards}

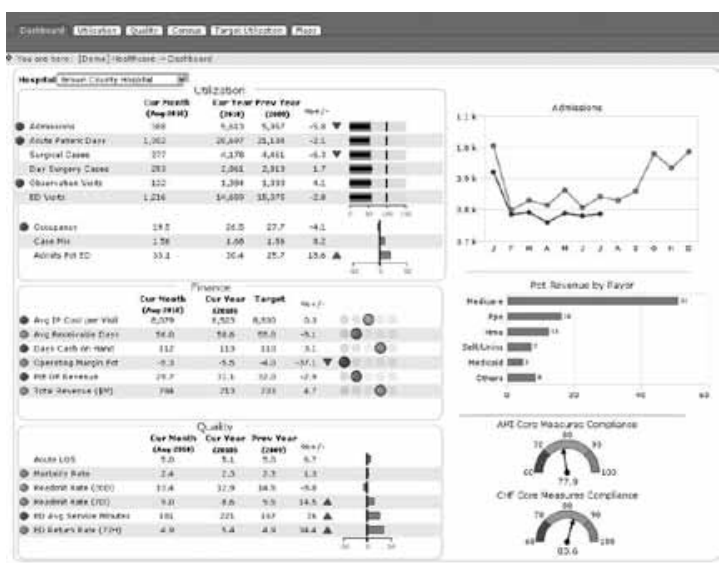




\section{TABLero de COMANDO EN EL SECTOR SALUd / E. MONTICO, M. VeLARde / 761}

\section{- "Semaforización" para una rápida identificación del estado del indicador}

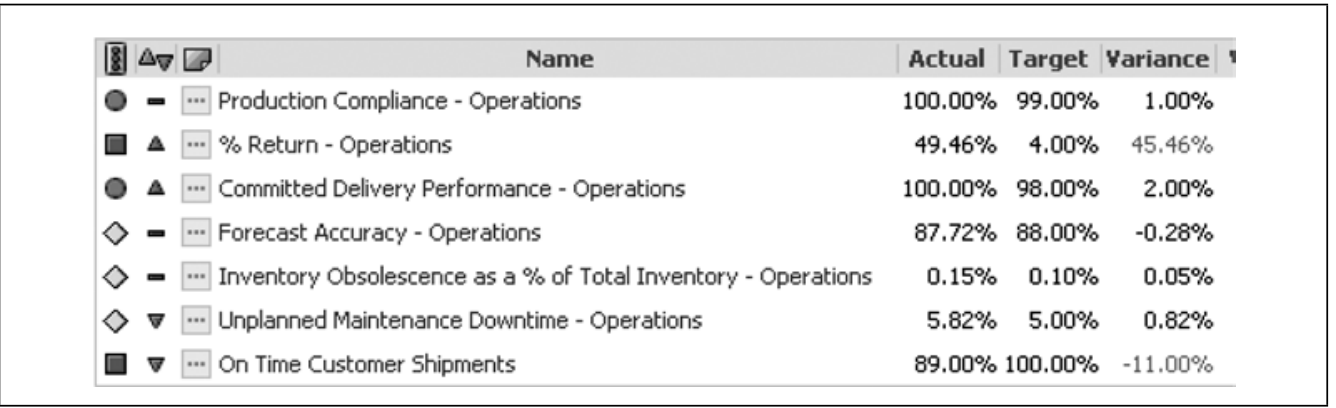

\section{- Diagramas de relación de causa y efecto}

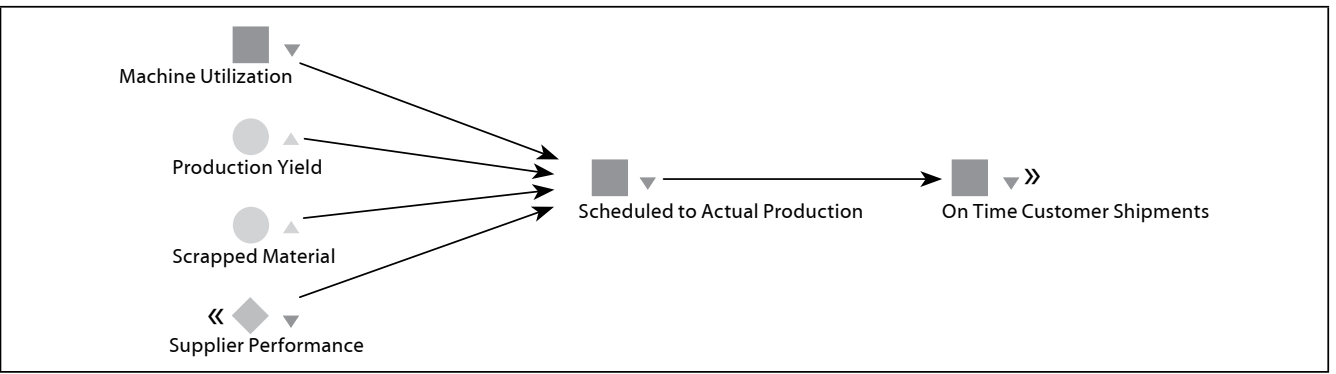

- Posibilidad de clasificar los indicadores de acuerdo a diversos criterios como las dimensiones habituales de un BSC (finanzas, clientes, procesos y aprendizaje) o muchas otras definidas directamente por el usuario.

- Posibilidad de interacción de los usuarios para incluir aclaraciones, notas y comentarios que permitan mejorar el entendimiento de los indicadores.

\section{2. ¿Por qué?}

Necesitamos entender los detalles de un resultado como punto de partida para poder corregirlo. Para esta etapa, podremos encontrar herramientas de análisis de mayor detalle y complejidad como:
- Reportes de soporte a los indicadores: pueden ser dinámicos para hacer un detalle sobre la información disponible mediante un "drill down" hacia las variables de cálculo del indicador y sus dimensiones de análisis.

- Análisis multidimensional: permiten la elaboración de modelos de información en distintas dimensiones de análisis (“cubos" de información). Una vez diseñado el modelo, este queda disponible para el usuario final de la herramienta, para así personalizar el criterio de análisis en función de las necesidades de información de cada usuario y de cada situación determinada.

- Análisis geográfico: la incorporación de cartografía permite el análisis por regiones, 
provincias o divisiones geográficas personalizadas como una dimensión adicional de análisis.

\section{3. ¿Qué debemos hacer?}

La planificación debe ser revisada y corregida para el logro de los objetivos; las herramientas nos permiten cubrir las distintas etapas de la planificación para modelar el negocio, al atar los drivers con resultados, promover un modelo colaborativo para garantizar el alineamiento y compromiso, y establecer así la base operacional.

A partir de este modelo de negocio, las herramientas informáticas aportan la posibilidad de aumentar la frecuencia y rapidez de la planificación, la presupuestación y sus revisiones periódicas; también brindan mayor precisión para reflejar rápidamente los cambios en los cursos de acción a partir de los resultados obtenidos.

\section{Referencias}

Carroll, Lewis, Alice's Adventures in Wonderland. John Tenniel (illustrator). London: MacMillan (1865).

Donabedian, Avedis (2002). An Introduction to Quality Assurance in Health Care. New York: Oxford University Press.

Guadalajara, Natividad (1994). Análisis de costes en los hospitales. Valencia: M/C/Q.

Kaplan, Robert \& Norton, David (1992). The Balanced Scorecard: Measures that Drive Performance. Harvard Business Review, JanuaryFebruary, 71-79. Disponible en: http://www.csee.umbc.edu/ sweet/Ph.D/ papers/BSC/BSC\%20-\%20Measures\%20 that $\% 20$ drive $\% 20$ performance.pdf

Montico-Riesco, Eduardo \& Velarde, Martín (2003). Una propuesta de costos para las instituciones de asistencia médica colectiva. Disponible en: http://www.monografias. com/trabajos14/costos-iamc/costos-iamc. shtml?monosearch

Neumann, John von \& Morgenstern, Oskar (1944). Theory of Games and Economic Behavior. Princeton, New Jersey: Princeton University Press.

World Health Organization, WHO (s.f.). Glossary. Disponible en: http://www.who.int/hia/ about/glos/en/, http://www.who.int/kobe_ centre/ageing/ahp_vol5_glossary.pdf, http:// www.who.int/healthsystems/hss_glossary/ en/index5.html, http://www.who.int/healthpromotion/about/HPG/en/

- Fecha de recepción: 1 de julio de 2014

- Fecha de aceptación: 30 de octubre de 2014

- Disponible en línea: 15 de diciembre de 2014

\section{Para citar este artículo}

Montico-Riesco, Eduardo \& Velarde, Martín (2014). Formulación de un tablero de comando para el análisis de la productividad y la gestión competitiva en el sector salud [número especial: Contabilidad Gerencial]. Cuadernos de Contabilidad, 15 (39), 745-762.

doi: 10.11144/Javeriana.cc15-39.ftca 